\title{
Fractional natural decomposition method for solving a certain class of nonlinear time-fractional wave-like equations with variable coefficients
}

Ali Khalouta

Laboratory of Fundamental and

Numerical Mathematics, Departement of Mathematics, Faculty of Sciences, Ferhat Abbas Sétif University 1, Algeria email: nadjibkh@yahoo.fr
Abdelouahab Kadem Laboratory of Fundamental and

Numerical Mathematics, Departement of Mathematics, Faculty of Sciences Ferhat Abbas Sétif University 1, Algeria email: abdelouahabk@yahoo.fr

\begin{abstract}
In this paper, we propose a new approximate method, namely fractional natural decomposition method (FNDM) in order to solve a certain class of nonlinear time-fractional wave-like equations with variable coefficients. The fractional natural decomposition method is a combined form of the natural transform method and the Adomian decomposition method. The nonlinear term can easily be handled with the help of Adomian polynomials which is considered to be a clear advantage of this technique over the decomposition method. Some examples are given to illustrate the applicability and the easiness of this approach.
\end{abstract}

\section{Introduction}

Fractional differential equations, as generalizations of classical integer order differential equations, are gradually employed to model problems in fluid flow, finance, physical, hydrological, biological processes and systems $[6,7,8,9]$.

2010 Mathematics Subject Classification: 35R11, 34K28, 26A33, 35A22

Key words and phrases: nonlinear time-fractional wave-like equations, Caputo fractional derivative, fractional natural decomposition method, Adomian polynomials 
The most frequent used methods for investigating fractional differential equations are: Adomian decomposition method (ADM) [1] variational iteration method (VIM) [12], generalized differential transform method (GDTM) [10], homotopy analysis method (HAM) [3], homotopy perturbation method (HPM) [11]. Also, there are some other classical solution techniques such as Laplace transform method, fractional Green's function method, Mellin transform method and method of orthogonal polynomials [8].

In this paper, the main objective is to solve a certain class of nonlinear timefractional wave-like equation with variable coefficients by using a modified method called fractional natural decomposition method (FNDM) which is a combination of two powerful methods, the Natural transform and the Adomian decomposition method.

Consider the following nonlinear time-fractional wave-like equations

$$
\begin{aligned}
D_{t}^{\alpha} v= & \sum_{i, j=1}^{n} F_{1 i j}(X, t, v) \frac{\partial^{k+m}}{\partial x_{i}^{k} \partial x_{j}^{m}} F_{2 i j}\left(v_{x_{i}}, v_{x_{j}}\right) \\
& +\sum_{i=1}^{n} G_{1 i}(X, t, v) \frac{\partial^{p}}{\partial x_{i}^{p}} G_{2 i}\left(v_{x_{i}}\right)+H(X, t, v)+S(X, t),
\end{aligned}
$$

with initial conditions

$$
v(X, 0)=a_{0}(X), v_{t}(X, 0)=a_{1}(X),
$$

where $D_{t}^{\alpha}$ is the Caputo fractional derivative operator of order $\alpha, 1<\alpha \leq 2$.

Here $X=\left(x_{1}, x_{2}, \ldots, x_{n}\right), F_{1 i j}, G_{1 i} i, j \in\{1,2, \ldots, n\}$ are nonlinear functions of $X, t$ and $v, F_{2 i j}, G_{2 i} i, j \in\{1,2, \ldots, n\}$, are nonlinear functions of derivatives of $v$ with respect to $x_{i}$ and $x_{j} i, j \in\{1,2, \ldots, n\}$, respectively. Also $H, S$ are nonlinear functions and $k, m, p$ are integers.

For $\alpha=2$, these types of equations are of considerable significance in various fields of applied sciences, mathematical physics, nonlinear hydrodynamics, engineering physics, biophysics, human movement sciences, astrophysics and plasma physics. These equations describe the evolution of erratic motions of small particles that are immersed in fluids, fluctuations of the intensity of laser light, velocity distributions of fluid particles in turbulent flows.

\section{Basic definitions}

In this section, we introduce some definitions and important properties of the fractional calculus, the natural transform, and the natural transform of fractional derivatives, which are used further in this paper. 


\subsection{Fractional calculus}

Definition 1 [8] A real function $\mathrm{f}(\mathrm{t}), \mathrm{t}>0$, is considered to be in the space $\mathrm{C}_{\mu}, \mu \in \mathbb{R}$ if there exists a real number $\mathrm{p}>\mu$, so that $\mathrm{f}(\mathrm{t})=\mathrm{t}^{\mathrm{p}} \mathrm{h}(\mathrm{t})$, where $\mathrm{h}(\mathrm{t}) \in \mathrm{C}\left(\left[0, \infty[)\right.\right.$, and it is said to be in the space $\mathrm{C}_{\mu}^{\mathrm{n}}$ if $\mathrm{f}^{(\mathrm{n})} \in \mathrm{C}_{\mu}, \mathrm{n} \in \mathbb{N}$.

Definition 2 [8] The Riemann-Liouville fractional integral operator $\mathrm{I}^{\alpha}$ of order $\alpha$ for a function $\mathrm{f} \in \mathrm{C}_{\mu}, \mu \geq-1$ is defined as follows

$$
I^{\alpha} f(t)= \begin{cases}\frac{1}{\Gamma(\alpha)} \int_{0}^{t}(t-\xi)^{\alpha-1} f(\xi) d \xi, & \alpha>0, t>0, \\ f(t), & \alpha=0,\end{cases}
$$

where $\Gamma($.$) is the well-known Gamma function.$

Definition 3 [8] The fractional derivative of $\mathbf{f}(\mathrm{t})$ in the Caputo sense is defined as follows

$$
D^{\alpha} f(t)=I^{n-\alpha} D^{n} f(t)=\frac{1}{\Gamma(n-\alpha)} \int_{0}^{t}(t-\xi)^{n-\alpha-1} f^{(n)}(\xi) d \xi, t>0,
$$

where $\mathrm{n}-1<\alpha \leq \mathrm{n}, \mathrm{n} \in \mathbb{N}, \mathrm{f} \in \mathrm{C}_{-1}^{\mathrm{n}}$.

For the Riemann-Liouville fractional integral and Caputo fractional derivative, we have the following relation

$$
I^{\alpha} D^{\alpha} f(t)=f(t)-\sum_{k=0}^{n-1} f^{(k)}\left(0^{+}\right) \frac{t^{k}}{k !}, t>0 .
$$

Definition 4 [8] The Mittag-Leffler function is defined as follows

$$
\mathrm{E}_{\alpha}(z)=\sum_{\mathrm{n}=0}^{\infty} \frac{z^{\mathrm{n}}}{\Gamma(\mathrm{n} \alpha+1)}, \alpha \in \mathbb{C}, \operatorname{Re}(\alpha)>0 .
$$

A further generalization of (6) is given in the form

$$
\mathrm{E}_{\alpha, \beta}(z)=\sum_{n=0}^{\infty} \frac{z^{\mathrm{n}}}{\Gamma(\mathrm{n} \alpha+\beta)}, \alpha, \beta \in \mathbb{C}, \operatorname{Re}(\alpha)>0, \operatorname{Re}(\beta)>0 .
$$

For $\alpha=1, \mathrm{E}_{\alpha}(z)$ reduces to $\mathrm{e}^{z}$. 


\subsection{Natural transform}

Definition 5 [2] The natural transform is defined over the set of functions is defined over the set of functions

$$
A=\left\{f(t) / \exists M, \tau_{1}, \tau_{2}>0,|f(t)|<M e^{\frac{|t|}{\tau_{j}}}, \text { if } t \in(-1)^{j} \times[0, \infty)\right\},
$$

by the following integral

$$
\mathcal{N}^{+}[\mathrm{f}(\mathrm{t})]=\mathrm{R}^{+}(\mathrm{s}, \mathrm{u})=\frac{1}{\mathrm{u}} \int_{0}^{+\infty} e^{-\frac{s t}{u}} f(t) d t, s, u \in(0, \infty) .
$$

Some basic properties of the natural transform are given as follows [2].

Property 1 The natural transform is a linear operator. That is, if $\lambda$ and $\mu$ are non-zero constants, then

$$
\mathcal{N}^{+}[\lambda f(t) \pm \mu g(t)]=\lambda \mathcal{N}^{+}[f(t)] \pm \mu \mathcal{N}^{+}[g(t)] .
$$

Property 2 If $f^{(n)}(t)$ is the $n$-th derivative of function $f(t)$ w.r.t. " $t$ " then its natural transform is given by

$$
\mathcal{N}^{+}\left[f^{(n)}(t)\right]=R_{n}^{+}(s, u)=\frac{s^{n}}{u^{n}} R^{+}(s, u)-\sum_{k=0}^{n-1} \frac{s^{n-(k+1)}}{u^{n-k}} f^{(k)}(0) .
$$

Property 3 (Convolution property) Suppose $\mathrm{F}^{+}(s, \mathrm{u})$ and $\mathrm{G}^{+}(\mathrm{s}, \mathrm{u})$ are the natural transforms of $f(t)$ and $g(t)$, respectively, both defined in the set $A$. Then the natural transform of their convolution is given by

$$
\mathcal{N}^{+}[(f * g)(t)]=u F^{+}(s, u) G^{+}(s, u),
$$

where the convolution of two functions is defined by

$$
(f * g)(t)=\int_{0}^{t} f(\xi) g(t-\xi) d \xi=\int_{0}^{t} f(t-\xi) g(\xi) d \xi .
$$

Property 4 Some special natural transforms

$$
\mathcal{N}^{+}[1]=\frac{1}{s}
$$




$$
\begin{aligned}
\mathcal{N}^{+}[\mathrm{t}] & =\frac{\mathrm{u}}{\mathrm{s}^{2}} \\
\mathcal{N}^{+}\left[\frac{\mathrm{t}^{\mathrm{n}-1}}{(\mathrm{n}-1) !}\right] & =\frac{\mathrm{u}^{\mathrm{n}-1}}{\mathrm{~s}^{\mathrm{n}}}, \mathrm{n}=1,2, \ldots
\end{aligned}
$$

Property 5 If $\alpha>-1$, then the natural transform of $t^{\alpha}$ is given by

$$
\mathcal{N}^{+}\left[\mathrm{t}^{\alpha}\right]=\Gamma(\alpha+1) \frac{\mathrm{u}^{\alpha}}{\mathrm{s}^{\alpha+1}} .
$$

\subsection{Natural transform of fractional derivatives}

Theorem 1 If $\mathrm{R}^{+}(\mathrm{s}, \mathrm{u})$ is the natural transform of $\mathrm{f}(\mathrm{t})$, then the natural transform of the Riemann-Liouville fractional integral for $\mathrm{f}(\mathrm{t})$ of order $\alpha$, is given by

$$
\mathcal{N}^{+}\left[I^{\alpha} f(t)\right]=\frac{u^{\alpha}}{s^{\alpha}} R^{+}(s, u) .
$$

Proof. The Riemann-Liouville fractional integral for the function $f(t)$, as in (3), can be expressed as the convolution

$$
I^{\alpha} f(t)=\frac{1}{\Gamma(\alpha)} t^{\alpha-1} * f(t)
$$

Applying the natural transform in the Eq. (10) and using Properties 3 and 5 , we have

$$
\begin{aligned}
\mathcal{N}^{+}\left[I^{\alpha} f(t)\right] & =\mathcal{N}^{+}\left[\frac{1}{\Gamma(\alpha)} t^{\alpha-1} * f(t)\right]=u \frac{1}{\Gamma(\alpha)} \mathcal{N}^{+}\left[t^{\alpha-1}\right] \mathcal{N}^{+}[f(t)] \\
& =u \frac{u^{\alpha-1}}{s^{\alpha}} R^{+}(s, u)=\frac{u^{\alpha}}{s^{\alpha}} R^{+}(s, u) .
\end{aligned}
$$

The proof is complete.

Theorem $2 \mathrm{n} \in \mathbb{N}^{*}$ and $\alpha>0$ be such that $\mathrm{n}-1<\alpha \leq \mathrm{n}$ and $\mathrm{R}^{+}(\mathrm{s}, \mathrm{u})$ be the natural transform of the function $\mathrm{f}(\mathrm{t})$, then the natural transform denoted by $\mathrm{R}_{\alpha}^{+}(\mathrm{s}, \mathrm{u})$ of the Caputo fractional derivative of the function $\mathrm{f}(\mathrm{t})$ of order $\alpha$, is given by

$$
\mathcal{N}^{+}\left[D^{\alpha} f(t)\right]=R_{\alpha}^{+}(s, u)=\frac{s^{\alpha}}{u^{\alpha}} R^{+}(s, u)-\sum_{k=0}^{n-1} \frac{s^{\alpha-(k+1)}}{u^{\alpha-k}}\left[D^{k} f(t)\right]_{t=0} .
$$


Proof. Let $g(t)=f^{(n)}(t)$, then by the Definition 3 of the Caputo fractional derivative, we obtain

$$
\begin{aligned}
D^{\alpha} f(t) & =\frac{1}{\Gamma(n-\alpha)} \int_{0}^{t}(t-\xi)^{n-\alpha-1} f^{(n)}(\xi) d \xi \\
& =\frac{1}{\Gamma(n-\alpha)} \int_{0}^{t}(t-\xi)^{n-\alpha-1} g(\xi) d \xi \\
& =I^{n-\alpha} g(t) .
\end{aligned}
$$

Applying the natural transform on both sides of (12) using Eq. (9), we get

$$
\mathcal{N}^{+}\left[D^{\alpha} f(t)\right]=\mathcal{N}^{+}\left[I^{n-\alpha} g(t)\right]=\frac{u^{n-\alpha}}{s^{n-\alpha}} G^{+}(s, u) .
$$

Also, we have from the Property 2

$$
\begin{aligned}
& \mathcal{N}^{+}[g(t)]=\mathcal{N}^{+}\left[f^{(n)}(t)\right], \\
& G^{+}(s, u)=\frac{s^{n}}{u^{n}} R^{+}(s, u)-\sum_{k=0}^{n-1} \frac{s^{n-(k+1)}}{u^{n-k}}\left[f^{(k)}(t)\right]_{t=0} .
\end{aligned}
$$

Hence, 13 becomes

$$
\begin{aligned}
\mathcal{N}^{+}\left[D^{\alpha} f(t)\right] & =\frac{u^{n-\alpha}}{s^{n-\alpha}}\left(\frac{s^{n}}{u^{n}} R^{+}(s, u)-\sum_{k=0}^{n-1} \frac{s^{n-(k+1)}}{u^{n-k}} f^{(k)}(0)\right) \\
& =\frac{s^{\alpha}}{u^{\alpha}} R^{+}(s, u)-\sum_{k=0}^{n-1} \frac{s^{\alpha-(k+1)}}{u^{\alpha-k}}\left[D^{k} f(t)\right]_{t=0}=R_{\alpha}^{+}(s, u), \\
-1 & <n-1<\alpha \leq n .
\end{aligned}
$$

The proof is complete.

\section{$3 \quad$ FNDM of nonlinear time-fractional wave-like equa- tions with variable coefficients}

Theorem 3 Consider the following nonlinear time-fractional wave-like equations (1) with the initial conditions (2). 
Then, by FNDM, the solution of Eqs. (1)-(2) is given in the form of infinite series as follows

$$
v(X, t)=\sum_{n=0}^{\infty} v_{n}(X, t) .
$$

Proof. In order to to achieve our goal, we consider the following nonlinear time-fractional wave-like equations (1) with the initial conditions (2).

First we define

$$
\begin{aligned}
& \mathrm{N} v=\sum_{i, j=1}^{n} F_{1 i j}(X, t, v) \frac{\partial^{k+m}}{\partial x_{i}^{k} \partial x_{j}^{m}} F_{2 i j}\left(v_{x_{i}}, v_{x_{j}}\right), \\
& M v=+\sum_{i=1}^{n} G_{1 i}(X, t, v) \frac{\partial^{p}}{\partial x_{i}^{p}} G_{2 i}\left(v_{x_{i}}\right), \\
& K v=H(X, t, v) .
\end{aligned}
$$

Eq. (1) is written in the form

$$
\begin{aligned}
D_{t}^{\alpha} v(X, t) & =N v(X, t)+M v(X, t)+K v(X, t)+S(X, t) \\
t & >0,1<\alpha \leq 2 .
\end{aligned}
$$

Applying the natural transform on both sides of (16) and using the Theorem 2 , we get

$$
\begin{aligned}
\mathcal{N}^{+}[v(X, t)]= & \frac{u^{\alpha}}{s^{\alpha}} \sum_{k=0}^{n-1} \frac{s^{\alpha-(k+1)}}{u^{\alpha-k}}\left[D^{k} v(X, t)\right]_{t=0} \\
& +\frac{u^{\alpha}}{s^{\alpha}} \mathcal{N}^{+}[N v(X, t)+M v(X, t)+K v(X, t)+S(X, t)] .
\end{aligned}
$$

After that, let us take the inverse natural transform on both sides of (17) we have

$$
v(X, t)=\mathrm{L}(\mathrm{X}, \mathrm{t})+\mathcal{N}^{-1}\left(\frac{\mathrm{u}^{\alpha}}{\mathrm{s}^{\alpha}} \mathcal{N}^{+}[\mathrm{N} v(X, \mathrm{t})+\mathrm{M} v(\mathrm{X}, \mathrm{t})+\mathrm{K} v(\mathrm{X}, \mathrm{t})]\right),
$$

where $L(X, t)$ is a term arising from the source term and the prescribed initial conditions.

Now, we represent the solution in an infinite series form

$$
v(X, t)=\sum_{n=0}^{\infty} v_{n}(X, t),
$$


and the nonlinear terms can be decomposed as

$$
N v(X, t)=\sum_{n=0}^{\infty} A_{n}, M v(X, t)=\sum_{n=0}^{\infty} B_{n}, K v(X, t)=\sum_{n=0}^{\infty} C_{n},
$$

where $A_{n}, B_{n}$ and $C_{n}$ are Adomian polynomials [13], of $v_{0}, v_{1}, v_{2}, . ., v_{n}$, and it can be calculated by formula given below

$$
A_{n}=B_{n}=C_{n}=\frac{1}{n !} \frac{d^{n}}{d \lambda^{n}}\left[N\left(\sum_{i=0}^{\infty} \lambda^{i} v_{i}\right)\right]_{\lambda=0}, n=0,1,2, \ldots
$$

Using Eqs. (19) and (20), we can rewrite Eq. (18) as

$$
\sum_{n=0}^{\infty} v_{n}(X, t)=L(X, t)+\mathcal{N}^{-1}\left(\frac{u^{\alpha}}{s^{\alpha}} \mathcal{N}^{+}\left[\sum_{n=0}^{\infty} A_{n}+\sum_{n=0}^{\infty} B_{n}+\sum_{n=0}^{\infty} C_{n}\right]\right) .
$$

By comparing both sides of Eq. (22) we have the following relation

$$
\begin{aligned}
& v_{0}(X, t)=L(X, t), \\
& v_{1}(X, t)=\mathcal{N}^{-1}\left(\frac{u^{\alpha}}{s^{\alpha}} \mathcal{N}^{+}\left[A_{0}+B_{0}+C_{0}\right]\right), \\
& v_{2}(X, t)=\mathcal{N}^{-1}\left(\frac{u^{\alpha}}{s^{\alpha}} \mathcal{N}^{+}\left[A_{1}+B_{1}+C_{1}\right]\right), \\
& v_{3}(X, t)=\mathcal{N}^{-1}\left(\frac{u^{\alpha}}{s^{\alpha}} \mathcal{N}^{+}\left[A_{2}+B_{2}+C_{2}\right]\right),
\end{aligned}
$$

and so on.

In general the recursive relation is given by

$$
\begin{aligned}
v_{0}(X, t) & =L(X, t), \\
v_{n+1}(X, t) & =\mathcal{N}^{-1}\left(\frac{u^{\alpha}}{s^{\alpha}} \mathcal{N}^{+}\left[A_{n}+B_{n}+C_{n}\right]\right), n \geq 0 .
\end{aligned}
$$

Then, the solution of Eqs. (1)-(2) is given in the form of infinite series as follows

$$
v(X, t)=\sum_{n=0}^{\infty} v_{n}(X, t) .
$$

The proof is complete. 
Theorem 4 Let $\mathcal{B}$ be a Banach space, Then the series solution of the Eqs. (1)-(2) converges to $S \in \mathcal{B}$, if there exists $\gamma, 0<\gamma<1$ such that

$$
\left\|v_{\mathrm{n}}\right\| \leq \gamma\left\|v_{\mathrm{n}-1}\right\|, \forall \mathrm{n} \in \mathbb{N} .
$$

Proof. Define the sequences $S_{n}$ of partial sums of the series given by the recursive relation (24) as

$$
S_{n}(X, t)=v_{0}(X, t)+v_{2}(X, t)+v_{3}(X, t)+\ldots+v_{n}(X, t),
$$

and we need to show that $\left\{S_{n}\right\}$ are a Cauchy sequences in Banach space $\mathcal{B}$. For this purpose, we consider

$$
\left\|S_{n+1}-S_{n}\right\| \leq\left\|v_{n+1}\right\| \leq \gamma\left\|v_{n}\right\| \leq \gamma^{2}\left\|v_{n-1}\right\| \leq \ldots \leq \gamma^{n+1}\left\|v_{0}\right\| .
$$

For every $\mathrm{n}, \mathrm{m} \in \mathbb{N}, \mathrm{n} \geq \mathrm{m}$, by using (26) and triangle inequality successively, we have

$$
\begin{aligned}
\left\|S_{n}-S_{m}\right\| & =\left\|S_{m+1}-S_{m}+S_{m+2}-S_{m+1}+\ldots+S_{n}-S_{n-1}\right\| \\
& \leq\left\|S_{m+1}-S_{m}\right\|+\left\|S_{m+2}-S_{m+1}\right\|+\ldots+\left\|S_{n}-S_{n-1}\right\| \\
& \leq \gamma^{m+1}\left\|v_{0}\right\|+\gamma^{m+2}\left\|v_{0}\right\|+\ldots+\gamma^{n}\left\|v_{0}\right\| \\
& =\gamma^{m+1}\left(1+\gamma+\ldots+\gamma^{n-m-1}\right)\left\|v_{0}\right\| \\
& \leq \gamma^{m+1}\left(\frac{1-\gamma^{n-m}}{1-\gamma}\right)\left\|v_{0}\right\| .
\end{aligned}
$$

Since $0<\gamma<1$, so $11-\gamma^{\mathrm{n}-\mathrm{m}} \leq 1$ then

$$
\left\|S_{n}-S_{m}\right\| \leq \frac{\gamma^{m+1}}{1-\gamma}\left\|v_{0}\right\|
$$

Since $v_{0}$ is bounded, then

$$
\lim _{n, m}\left\|S_{n}-S_{m}\right\|=0 .
$$

Therefore, the sequences $\left\{S_{n}\right\}$ are Cauchy sequences in the Banach space $\mathcal{B}$, so the series solution defined in (25) converges. This completes the proof.

Remark 1 The m-term approximate solution of Eqs. (1)-(2) is given by

$$
v(X, t)=\sum_{n=0}^{m-1} v_{n}(X, t)=v_{0}(X, t)+v_{1}(X, t)+v_{2}(X, t)+\ldots
$$




\section{Appliquations and numerical results}

In this section, we apply the (FNDM) on three examples of nonlinear timefractional wave-like equations with variable coefficients and then compare our approximate solutions with the exact solutions.

Example 1 Consider the 2-dimensional nonlinear time-fractional wave-like equation with variable coefficients

$$
\mathrm{D}_{\mathrm{t}}^{\alpha} v=\frac{\partial^{2}}{\partial x \partial y}\left(v_{x x} v_{y y}\right)-\frac{\partial^{2}}{\partial x \partial y}\left(x y v_{x} v_{y}\right)-v, 1<\alpha \leq 2,
$$

with initial conditions

$$
v(x, y, 0)=e^{x y}, v_{t}(x, y, 0)=e^{x y},
$$

where $\mathrm{D}_{\mathrm{t}}^{\alpha}$ is the Caputo fractional derivative operator of order $\alpha$, and $v$ is a function of $(x, y, t) \in \mathbb{R}^{2} \times \mathbb{R}^{+}$.

By applying the steps involved in (FNDM) as presented in Section 3 to Eqs. (27)-(28), we have

$$
\begin{aligned}
& v_{0}(x, y, t)=(1+t) e^{x y}, \\
& v_{1}(x, y, t)=-\left(\frac{t^{\alpha}}{\Gamma(\alpha+1)}+\frac{t^{\alpha+1}}{\Gamma(\alpha+2)}\right) e^{x y} \text {, } \\
& v_{2}(x, y, t)=\left(\frac{t^{2 \alpha}}{\Gamma(2 \alpha+1)}+\frac{t^{2 \alpha+1}}{\Gamma(2 \alpha+2)}\right) e^{x y},
\end{aligned}
$$

So, the solution of Eqs. (27)-(28) can be expressed by

$$
\begin{aligned}
v(x, y, t) & =\sum_{n=0}^{\infty} v_{n}(x, y, t) \\
& =\left(1+t-\frac{t^{\alpha}}{\Gamma(\alpha+1)}-\frac{t^{\alpha+1}}{\Gamma(\alpha+2)}+\frac{t^{2 \alpha}}{\Gamma(2 \alpha+1)}+\frac{t^{2 \alpha+1}}{\Gamma(2 \alpha+2)}-\ldots\right) e^{x y} . \\
& =\left(E_{\alpha}\left(-t^{\alpha}\right)+t E_{\alpha, 2}\left(-t^{\alpha}\right)\right) e^{x y},
\end{aligned}
$$

where $\mathrm{E}_{\alpha}\left(-\mathrm{t}^{\alpha}\right) \mathrm{e}^{\mathrm{xy}}$ and $\mathrm{E}_{\alpha, 2}\left(-\mathrm{t}^{\alpha}\right)$ are the Mittag-Leffler functions, defined by Eqs. (6) and (7).

Taking $\alpha=2$ in (29), the solution of Eqs. (27)-(28) has the general pattern form which is coinciding with the following exact solution in terms of infinite series

$$
v(x, y, t)=\left(1+t-\frac{t^{2}}{2 !}-\frac{t^{3}}{3 !}+\frac{t^{4}}{4 !}+\frac{t^{5}}{5 !}-\ldots\right) e^{x y} .
$$


So, the exact solution of Eqs. (27)-(28) in a closed form of elementary function will be

$$
v(x, y, t)=(\cos t+\sin t) e^{x y},
$$

which is the same result obtained by (ADM) [4] and (HPTM) [5], for the same test problem.

(a)

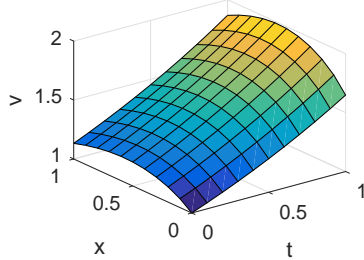

(c)

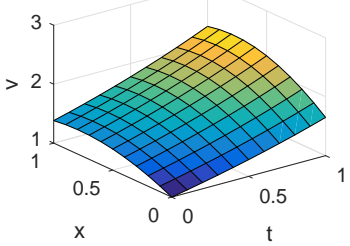

(b)

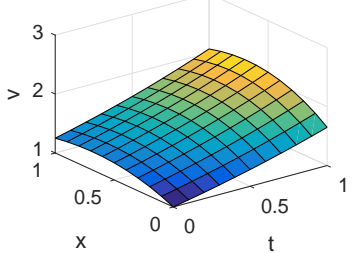

(d)

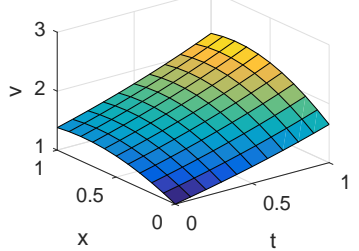

Figure 1: The surface graph of the 4-term approximate solution by (FNDM) and the exact solution for Example 1 when $y=0.5$ : (a) $v$ when $\alpha=1.5$, (b) $v$ when $\alpha=1.75$, (c) $v$ when $\alpha=2$, and (d) $v$ exact.

\begin{tabular}{|c|c|c|c|c|c|c|}
\hline $\mathrm{t}$ & $\alpha=1.7$ & $\alpha=1.8$ & $\alpha=1.95$ & $\alpha=2$ & exact solution & $\left|v_{\text {exact }}-v_{\text {FNDM }}\right|$ \\
\hline 0.1 & 1.3953 & 1.3999 & 1.4046 & 1.4058 & 1.4058 & $3.2196 \times 10^{-13}$ \\
0.3 & 1.5522 & 1.5735 & 1.5991 & 1.6061 & 1.6061 & $2.1569 \times 10^{-9}$ \\
0.5 & 1.6359 & 1.6755 & 1.7272 & 1.7424 & 1.7424 & $1.3095 \times 10^{-7}$ \\
0.7 & 1.6540 & 1.7088 & 1.7854 & 1.8093 & 1.8093 & $1.9680 \times 10^{-6}$ \\
0.9 & 1.6137 & 1.6775 & 1.7728 & 1.8040 & 1.8040 & $1.4947 \times 10^{-5}$ \\
\hline
\end{tabular}

Table 1: The numerical values of the 4-term approximate solution and the exact solution for Example 1 when $x=y=0.5$. 


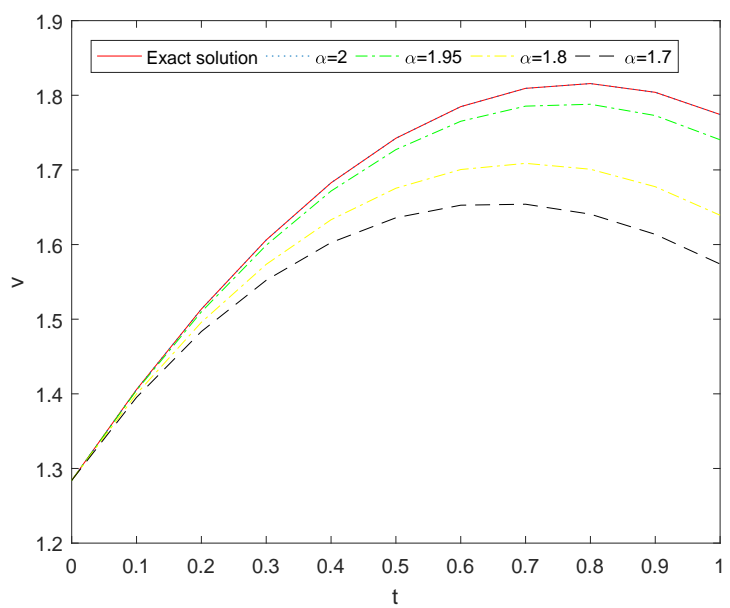

Figure 2: The behavior of the 4-term approximate solution by (FNDM) and the exact solution for Example 1 for different values of $\alpha$ when $x=y=0.5$.

Example 2 Consider the following nonlinear time-fractional wave-like equation with variable coefficients

$$
\mathrm{D}_{\mathrm{t}}^{\alpha} v=v^{2} \frac{\partial^{2}}{\partial x^{2}}\left(v_{x} v_{x x} v_{x x x}\right)+v_{x}^{2} \frac{\partial^{2}}{\partial x^{2}}\left(v_{x x}^{3}\right)-18 v^{5}+v, 1<\alpha \leq 2,
$$

with initial conditions

$$
v(x, 0)=e^{x}, v_{t}(x, 0)=e^{x},
$$

where $\mathrm{D}_{\mathrm{t}}^{\alpha}$ is the Caputo fractional derivative operator of order $\alpha$, and $v$ is a function of $(x, t) \in] 0,1\left[\times \mathbb{R}^{+}\right.$.

By applying the steps involved in (FNDM) as presented in Section 3 to Eqs. (30)-(31), we have

$$
\begin{gathered}
v_{0}(x, t)=(1+t) e^{x}, \\
v_{1}(x, t)=\left(\frac{t^{\alpha}}{\Gamma(\alpha+1)}+\frac{t^{\alpha+1}}{\Gamma(\alpha+2)}\right) e^{x}, \\
v_{2}(x, t)=\left(\frac{t^{2 \alpha}}{\Gamma(2 \alpha+1)}+\frac{t^{2 \alpha+1}}{\Gamma(2 \alpha+2)}\right) e^{x}, \\
\ldots
\end{gathered}
$$


So, the solution of Eqs. (30)-(31) can be expressed by

$$
\begin{aligned}
v(x, t) & =\left(1+t+\frac{t^{\alpha}}{\Gamma(\alpha+1)}+\frac{t^{\alpha+1}}{\Gamma(\alpha+2)}+\frac{t^{2 \alpha}}{\Gamma(2 \alpha+1)}+\frac{t^{2 \alpha+1}}{\Gamma(2 \alpha+2)}+\ldots\right) e^{x} \\
& =\left(E_{\alpha}\left(t^{\alpha}\right)+t E_{\alpha, 2}\left(t^{\alpha}\right)\right) e^{x}
\end{aligned}
$$

where $\mathrm{E}_{\alpha}\left(\mathrm{t}^{\alpha}\right)$ and $\mathrm{E}_{\alpha, 2}\left(\mathrm{t}^{\alpha}\right)$ are the Mittag-Leffler functions, defined by Eqs. (6) and (7).

Taking $\alpha=2$ in (32), the solution of Eqs. (30)-(31) has the general pattern form which is coinciding with the following exact solution in terms of infinite series

$$
v(x, t)=\left(1+t+\frac{t^{2}}{2 !}+\frac{t^{3}}{3 !}+\frac{t^{4}}{4 !}+\frac{t^{5}}{5 !}+\ldots\right) e^{x} .
$$

So, the exact solution of Eqs. (30)-(31) in a closed form of elementary function will be

$$
v(x, t)=e^{x+t},
$$

which is the same result obtained by (ADM) [4] and (HPTM) [5], for the same test problem.

(a)

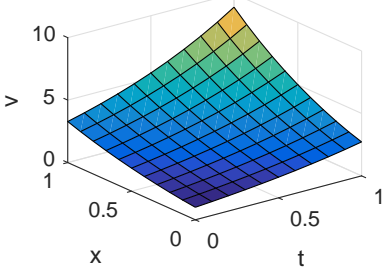

(c)

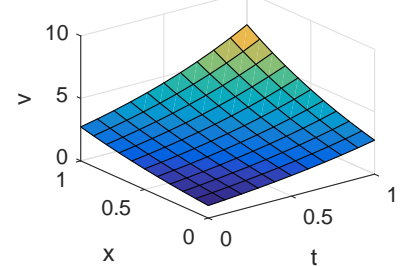

(b)

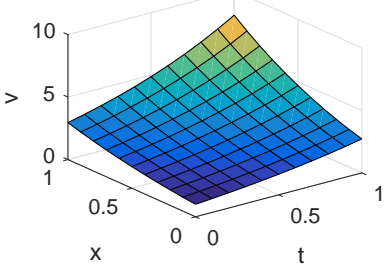

(d)

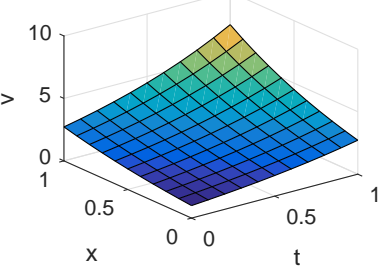

Figure 3: The surface graph of the 4-term approximate solution by (FNDM) and the exact solution for Example 2: (a) $v$ when $\alpha=1.5$, (b) $v$ when $\alpha=1.75$, (c) $v$ when $\alpha=2$, and (d) $v$ exact. 


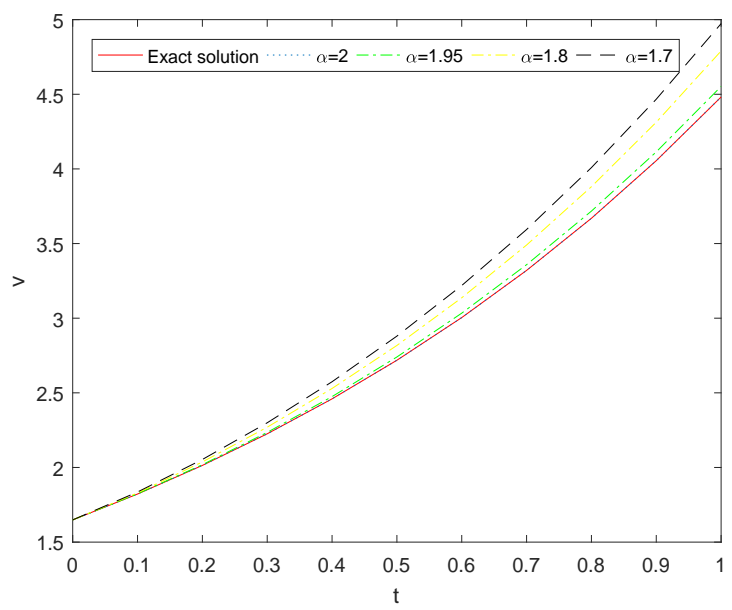

Figure 4: The behavior of the 4-term approximate solution by (FNDM) and the exact solution for Example 2 for different values of $\alpha$ when $x=0.5$.

\begin{tabular}{|c|c|c|c|c|c|c|}
\hline $\mathrm{t}$ & $\alpha=1.7$ & $\alpha=1.8$ & $\alpha=1.95$ & $\alpha=2$ & exact solution & $\left|v_{\text {exact }}-v_{\text {FNDM }}\right|$ \\
\hline 0.1 & 1.8357 & 1.8298 & 1.8236 & 1.8221 & 1.8221 & $4.1350 \times 10^{-13}$ \\
0.3 & 2.2994 & 2.2697 & 2.2350 & 2.2255 & 2.2255 & $2.7750 \times 10^{-9}$ \\
0.5 & 2.8800 & 2.8174 & 2.7402 & 2.7183 & 2.7183 & $1.6907 \times 10^{-7}$ \\
0.7 & 3.5940 & 3.4901 & 3.3585 & 3.3201 & 3.3201 & $2.5543 \times 10^{-6}$ \\
0.9 & 4.4670 & 4.3129 & 4.1140 & 4.0552 & 4.0552 & $1.9535 \times 10^{-5}$ \\
\hline
\end{tabular}

Table 2: The numerical values of the 4-term approximate solution and the exact solution for Example 2 when $x=0.5$.

Example 3 Consider the following one dimensional nonlinear time-fractional wave-like equation with variable coefficients

$$
\mathrm{D}_{\mathrm{t}}^{\alpha} v=x^{2} \frac{\partial}{\partial x}\left(v_{x} v_{x x}\right)-x^{2}\left(v_{x x}\right)^{2}-v, 1<\alpha \leq 2,
$$

with initial conditions

$$
v(x, 0)=0, v_{t}(x, 0)=x^{2},
$$

where $\mathrm{D}_{\mathrm{t}}^{\alpha}$ is the Caputo fractional derivative operator of order $\alpha$, and $v$ is a function of $(x, t) \in] 0,1\left[\times \mathbb{R}^{+}\right.$. 
By applying the steps involved in (FNDM) as presented in Section 3 to Eqs. (33)-(34), we have

$$
\begin{gathered}
v_{0}(x, t)=t x^{2} \\
v_{1}(x, t)=-\frac{t^{\alpha+1}}{\Gamma(\alpha+2)} x^{2}, \\
v_{2}(x, t)=\frac{t^{2 \alpha+1}}{\Gamma(2 \alpha+2)} x^{2} \\
\ldots
\end{gathered}
$$

So, the solution of Eqs. (33)-(34) can be expressed by

$$
\begin{aligned}
v(x, t) & =\sum_{n=0}^{\infty} v_{n}(x, t) \\
& =x^{2}\left(t-\frac{t^{\alpha+1}}{\Gamma(\alpha+2)}+\frac{t^{2 \alpha+1}}{\Gamma(2 \alpha+2)}-\ldots\right) \\
& =x^{2}\left(\mathrm{tE}_{\alpha, 2}\left(-\mathrm{t}^{\alpha}\right)\right),
\end{aligned}
$$

where $\mathrm{E}_{\alpha, 2}\left(-\mathrm{t}^{\alpha}\right)$ is the Mittag-Leffler function, defined by Eq. (6).

Taking $\alpha=2$ in (35), the solution of Eqs. (33)-(34) has the general pattern form which is coinciding with the following exact solution in terms of infinite series

$$
v(x, t)=x^{2}\left(t-\frac{t^{3}}{3 !}+\frac{t^{5}}{5 !}-\ldots\right) .
$$

So, the exact solution of Eqs. (33)-(34) in a closed form of elementary function will be

$$
v(x, t)=x^{2} \sin t,
$$

which is the same result obtained by (ADM) [4] and (HPTM) [5], for the same test problem.

Remark 2 The numerical results (See Figures 1, 2,.., 6) and (Tables 1, 2 and 3$)$, affirm that when $\alpha$ approaches 2 , our results approach the exact solutions.

Remark 3 In this paper, we only apply four terms to approximate the solutions, if we apply more terms of the approximate solutions, the accuracy of the approximate solutions will be greatly improved. 
(a)

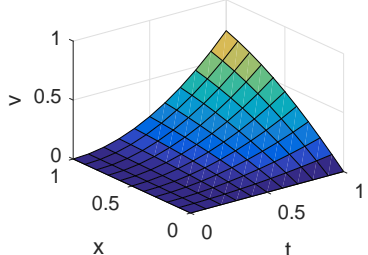

(c)

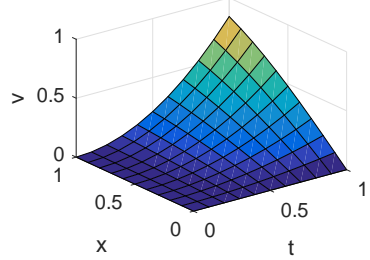

(b)

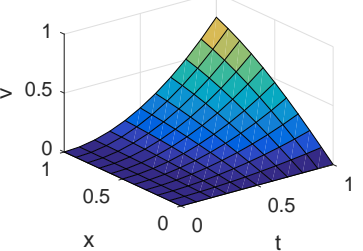

(d)

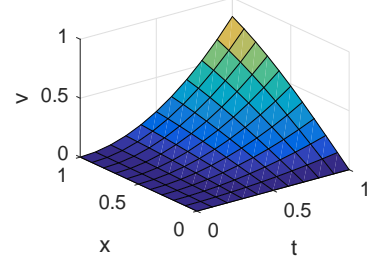

Figure 5: The surface graph of the 4-term approximate solution by (FNDM) and the exact solution for Example 3: (a) $v$ when $\alpha=1.5$, (b) $v$ when $\alpha=1.75$, (c) $v$ when $\alpha=2$, and (d) $v$ exact.

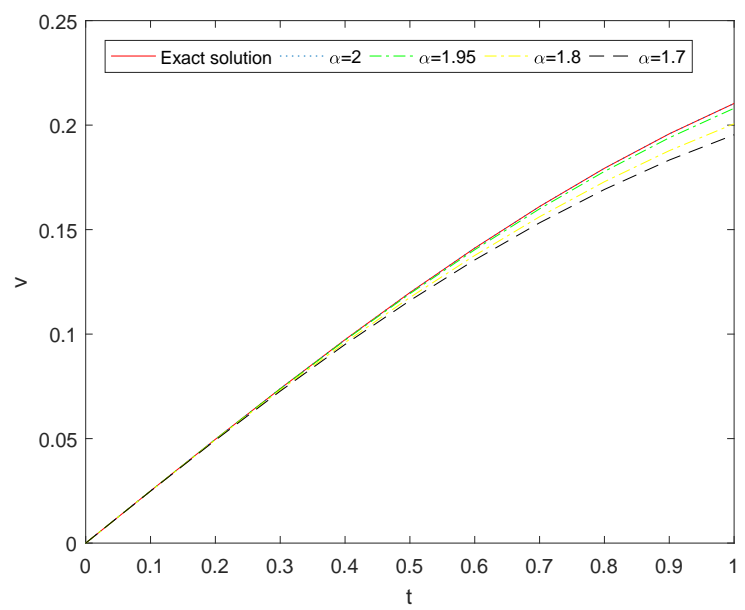

Figure 6: The behavior of the 4-term approximate solution by (FNDM) and the exact solution for Example 3 for different values of $\alpha$ when $x=0.5$. 


\begin{tabular}{|c|c|c|c|c|c|c|}
\hline $\mathrm{t}$ & $\alpha=1.7$ & $\alpha=1.8$ & $\alpha=1.95$ & $\alpha=2$ & exact solution & $\left|v_{\text {exact }}-v_{\text {FNDM }}\right|$ \\
\hline 0.1 & 0.02488 & 0.02492 & 0.02495 & 0.02496 & 0.02496 & $6.8887 \times 10^{-16}$ \\
0.3 & 0.07271 & 0.07319 & 0.07374 & 0.07388 & 0.07388 & $1.3549 \times 10^{-11}$ \\
0.5 & 0.11604 & 0.11752 & 0.11934 & 0.11986 & 0.11986 & $1.3425 \times 10^{-9}$ \\
0.7 & 0.15325 & 0.15615 & 0.15994 & 0.16105 & 0.16105 & $2.7677 \times 10^{-8}$ \\
0.9 & 0.18327 & 0.18777 & 0.19394 & 0.19583 & 0.19583 & $2.6495 \times 10^{-7}$ \\
\hline
\end{tabular}

Table 3: The numerical values of the 4-term approximate solution and the exact solution for Example 3 when $x=0.5$.

\section{Conclution}

In this paper, the (FNDM) has been successfully applied to study a certain class of nonlinear time-fractional wave-like equations with variable coefficients. The results show that the (FNDM) is an efficient and easy to use technique for finding approximate and exact solutions for this equation. The obtained approximate solutions using the suggested method is in excellent agreement with the exact solution. This confirms our belief that the efficiency of our technique gives it much wider applicability for general classes of nonlinear problems.

\section{Acknowledgment}

The authors are very grateful to the referees for carefully reading the paper and for their important remarks and suggestions which have improved the paper.

\section{References}

[1] S. Abbasbandy, Homotopy perturbation method for quadratic Riccati differential equation and comparison with Adomians decomposition method, Appl. Math. Comput., 172 (1) (2006), 485-490.

[2] F. B. M. Belgacem, R. Silambarasan, Theory of natural transform, Math. Eng. Sci. Aerosp., 3 (1) (2012), 105-135.

[3] M. Ganjiani, Solution of nonlinear fractional differential equations using homotopy analysis method, Appl. Math. Model., 34 (2010), 1634-1641. 
[4] M. Ghoreishi, A.I. B. Ismail, N.H. M. Ali, Adomain decomposition method for nonlinear wave- like equation with variable coefficients, Appl. Math. Sci., 4 (49) (2010), 2431-2444.

[5] V. G. Gupta, S. Gupta, Homotopy perturbation transform method for solving nonlinear wave- like equations of variable coefficients, J. Inf. Comput. Sci., 8 (3) (2013), 163-172.

[6] A. A Kilbas, H. M Srivastava, J. J. Trujillo, Theory and Applications of Fractional Differential Equations, Elsevier, Amsterdam, 2006.

[7] K. S. Miller, B. Ross, An Introduction to the Fractional Calculus and Fractional Differential Equations, Wiley, New York, 1993.

[8] I. Podlubny, Fractional Differential Equations, Academic Press, New York, 1999.

[9] I. Podlubny, Geometric and physical interpretation of fractional integration and fractional differentiation, Fract. Calc. Appl. Anal., 5 (2002), $367-386$.

[10] Z. Odibat, S. Momani, A generalized differential transform method for linear partial differential equations of fractional order, Appl. Math. Lett., 21 (2008), 194-199.

[11] Z. Odibat, S. Momani, Modified homotopy perturbation method: application to quadratic Riccati differential equation of fractional order, Chaos Solitons Fractals, 36 (1) (2008), 167-174.

[12] Y. Zhang, Time-Fractional Generalized Equal Width Wave Equations: Formulation and Solution via Variational Methods, Nonlinear Dyn. Syst. Theory, 14 (4) (2014), 410-425.

[13] Y. Zhu, Q. Chang, S. Wu, A new algorithm for calculating Adomian polynomials, Appl. Math. Comput., 169 (2005), 402-416. 